\title{
Perfil de gestantes adolescentes e adultas jovens acompanhadas por uma Unidade de Saúde da Família
}

\author{
Profile of pregnant adolescents and young adults, accompanied by a Family Health Unit
}

Perfil de las adolescentes y jóvenes embarazadas, acompañadas de una Unidad de Salud de la Familia

Marcos Antônio Sales Rodrigues ${ }^{1 *}$, Kátia Fernanda Alves Moreira1, Daiana Evangelista Rodrigues Fernandes ${ }^{1}$, Aldrin de Sousa Pinheiro ${ }^{1}$, Nayra Carla de Melo ${ }^{1}$, Jandra Cibele Rodrigues de Abrantes Pereira Leite ${ }^{1}$.

\section{RESUMO}

Objetivo: Investigar o perfil sociodemográfico, reprodutivo e obstétrico em gestantes adolescentes e adultas jovens acompanhadas pelas equipes de saúde da família matutinas de uma Unidade Básica Saúde. Métodos: Estudo quantitativo, transversal, com 39 gestantes acompanhadas por equipes da família matutinas em uma Unidade de Saúde de um município do estado de Rondônia. A amostra foi por conveniência. Os dados foram analisados por meio de estatística descritiva. Resultados: A maioria das gestantes possui idade entre 15 e 19 anos (56,41\%), casadas ou em união estável (82,05\%). A maior parte tornou-se sexualmente ativa na faixa etária de 15 a 19 anos (53,85\%); utilizam métodos de barreira como forma de prevenção de infeções sexualmente transmissíveis $(53,85 \%)$ e gestações não planejadas $(92,31 \%)$. Foram referenciadas para serviço de alto risco, 33,33\% e buscam a maternidade como unidade referência em casos de emergências obstétricas (55,85\%). Conclusão: A maior parte das gestantes se encontra em situação de vulnerabilidade social e sexual/reprodutiva, o qual reflete na busca tardia aos serviços pré-natais e menor adesão às condutas estabelecidas ao longo do período gestacional e prevenção de possíveis intercorrências gestacionais. Evidenciou-se barreiras e empecilhos no acesso aos serviços de saúde de pré-natal.

Palavras-chave: Gravidez na adolescência, Cuidado pré-natal, Saúde sexual e reprodutiva, Gravidez não planejada, Atenção primária à saúde.

\begin{abstract}
Objective: To investigate the sociodemographic, reproductive and obstetric profile of pregnant adolescents and young adults accompanied by morning family health teams at a Basic Health Unit. Methods: Quantitative, cross-sectional study with 39 pregnant women accompanied by morning family teams in a Health Unit of a municipality in state of Rondônia. The sample was for convenience. Data were analyzed using descriptive statistics. Results: Most pregnant women are between 15 and 19 years old (56.41\%), married or in a stable relationship (82.05\%). Most became sexually active in the age group of 15 to 19 years (53.85\%); use barrier methods as a way to prevent sexually transmitted infections (53.85\%) and unplanned pregnancies (92.31\%). 33.33\% were referred to a high-risk service and sought the maternity hospital as a reference unit in cases of obstetric emergencies (55.85\%). Conclusion: Most pregnant women are in a situation of social and sexual/reproductive vulnerability, which reflects on the late search for prenatal services and lower adherence to the behaviors established throughout the gestational period and prevention of possible gestational complications. Barriers and obstacles to access to prenatal health services were evidenced.
\end{abstract}

Keywords: Adolescent pregnancy, Prenatal care, Sexual and reproductive health, Unplanned pregnancy, Primary health care.

1 Universidade Federal de Rondônia (UNIR), Porto Velho - RO. *E-mail: marcos.sls.rodrigues@gmail.com 


\section{RESUMEN}

Objetivo: Investigar el perfil sociodemográfico, reproductivo y obstétrico de adolescentes y jóvenes embarazadas acompañadas por equipos matutinos de salud de la familia en una Unidad Básica de Salud. Métodos: Estudio cuantitativo, transversal con 39 gestantes acompañadas por equipos matutinos de salud familiar en una Unidad de Salud de un municipio del estado de Rondônia. La muestra fue por conveniencia. Los datos fueron analizados utilizando estadística descriptiva. Resultados: La mayoría de las gestantes tienen entre 15 y 19 años (56,41\%), casadas o en pareja estable (82,05\%). La mayoría inició su actividad sexual en el grupo de edad de 15 a 19 años (53,85\%); utilizan métodos de barrera como forma de prevención de infecciones de transmisión sexual $(53,85 \%)$ y embarazos no planificados $(92,31 \%)$. El $33,33 \%$ fueron derivadas a un servicio de alto riesgo y buscaron la maternidad como unidad de referencia en casos de urgencias obstétricas $(55,85 \%)$. Conclusión: La mayoría de las gestantes se encuentran en situación de vulnerabilidad social y sexual/reproductiva, lo que se refleja en la búsqueda tardía de servicios de prenatal y menor adherencia a las conductas establecidas a lo largo del período gestacional y prevención de posibles complicaciones gestacionales. Se evidenciaron barreras y obstáculos para el acceso a los servicios de salud prenatal.

Palabras clave: Embarazo adolescente, Atención prenatal, Salud sexual y reproductiva, Embarazo no planificado, Atención primaria de salud.

\section{INTRODUÇÃO}

Adolescência e a juventude são momentos que marcam a saída da infância para fase adulta, sendo a adolescência caracterizada pela faixa etária dos 10 aos 19 anos e os adultos jovens, a população de 15 a 24 anos (MINISTÉRIO DA SAÚDE, 2010; MINISTÉRIO DA SAÚDE, 2018). Essa faixa etária é vulnerável a grandes problemáticas de cunho social como práticas sexuais inseguras, o uso e abuso de drogas e a gestação não planejada. Além disso, tais questões sociais são evidenciadas em populações economicamente desfavorecidas e, portanto, mais suscetíveis a desdobramento negativos que podem impactar suas vidas em longo prazo (JACOB DSG, et al., 2020; CINTRA LCG, et al., 2020).

A gravidez não planejada principalmente na adolescência se configura como uma problemática de saúde pública, principalmente em países emergentes, que se sustentam sobre os estigmas relacionados à educação sexual em ambientes familiares e escolares, com forte influência das questões de gênero e sem o preparo psicológico, social e econômico, colocando o binômio mãe-criança em vulnerabilidade social (SILVA MRB, et al., 2019; FERNANDES DER, et al., 2020).

Desse modo a ocorrência da gravidez não intencional no público jovem em contextos sociais e econômicos desfavoráveis, se configura como um dos principais problemas de saúde pública em todo o mundo, principalmente em nações emergentes. De acordo com a OMS, sete países constituem principalmente a metade de todas as gestações não planejadas em adolescentes, a saber, Bangladesh, Brasil, República Democrática do Congo, Etiópia, Índia, Nigéria e Estados Unidos da América (PLUMMER ML, et al., 2017; FERNANDES DER, et al., 2020).

No Brasil, estudos no início dos anos 2000, já demonstravam que a gestação na adolescência estava associada com condições de vida socioeconômicas mais baixas como menos anos de estudos, baixa renda e pouca assistência ao pré-natal, quando em comparação com a faixa etária de mulheres adultas jovens. A região Norte do Brasil ainda possui as maiores taxas de gestantes adolescentes. Quando observados os fatores de risco reprodutivos e obstétricos possuem os maiores números, devido a ser a faixa etária com maior número de fecundidade e natalidade (KASSAR SB, et al., 2006; CRUZ MS, et al., 2016; RODRIGUES ARM, et al., 2017).

No contexto da Atenção Primária à Saúde (APS) e Estratégia de Saúde da Família, as Unidades Básicas de Saúde (UBS) devem fornecer um ambiente acolhedor, privado e seguro centrado na pessoa para que adolescentes sexualmente ativos recebam serviços oportunos e métodos contraceptivos gratuitos para prevenir gravidezes indesejadas ou evitar possíveis complicações ao longo da gestação quando em acompanhamento gestacional (MARTINS MMF, et al., 2019). 
Portanto, o acesso aos cuidados pré-natais de qualidade, informações precisas e educação em saúde sobre os diferentes anticoncepcionais devem ser fornecidas aos adolescentes sexualmente ativos para que eles façam uma escolha informada o que pode ajudar a evitar abortos ou gravidez na adolescência, assim como a redução de morbimortalidade materna (PLUMMER ML, et al., 2017).

Diante dessas considerações iniciais, esse estudo objetivou investigar o perfil obstétrico, reprodutivo, sociodemográfico de gestantes adolescentes e adultas jovens acompanhadas pelas equipes de saúde da família matutinas de uma Unidade Saúde da Família, o que pode caracterizar as condições de vida em que estão inseridas estas jovens mães.

\section{MÉTODOS}

Trata-se de um estudo quantitativo, descritivo, de corte transversal. A pesquisa foi realizada na zona Leste de um município do estado de Rondônia, com as gestantes adolescentes e adultas jovens acompanhadas pelas Equipes de Saúde da Família (ESF) do período da manhã, a qual foi norteada pela seguinte questão: Qual o perfil de gestantes adolescentes e jovens que realizam o pré-natal em uma equipe de saúde da família? A partir disso a amostra foi constituída por 39 gestantes adolescentes e adultas jovens, residentes na área de atuação das ESF, no período de setembro a dezembro/2021.

Critérios de inclusão: ser residente na área de abrangência da ESF ter realizado ou estar realizando o pré-natal na UBS, em sendo adolescente ter autorizado, por si ou por seus pais e/ou responsáveis e ter idade entre 10 e 24, visto que segundo os critérios adotados pelo Ministério da Saúde, tal faixa etária caracteriza a população de pessoas jovens, o que inclui adolescentes de 10 a 19 anos e adultos jovens de 20 a 24 anos (MINISTÉRIO DA SAÚDE, 2010; MINISTÉRIO DA SAÚDE, 2018).

Para os critérios de exclusão da amostra adotaram-se as gestantes que sofreram algum tipo de aborto e de áreas descobertas no território da UBS.

Para a coleta realizou-se um contato prévio com as enfermeiras da UBS, para o levantamento dessas gestantes. Obteve-se informações como nome, idade, data de nascimento, número de telefone, Cartão Nacional do SUS (CNS) e Agente Comunitário de Saúde (ACS) que cobre a microárea em que as gestantes residem. Posteriormente essas gestantes foram contatadas por meio de chamadas telefônicas e aplicativos de mensagens/conversas em tempo real, juntamente ao ACS.

Ao concordarem participar do estudo, o ACS levava o Termo de Consentimento Livre e Esclarecido (TCLE) e o Termo de Assentimento (se necessário) para serem assinados e entregues ao pesquisador na sexta-feira. Após essa condição, informada ao pesquisador pelo ACS, iniciava-se de forma remota a aplicação dos questionários.

O questionário foi composto por blocos de variáveis divididas em questões socioeconômicas, sobre a vida sexual e reprodutiva; sobre características obstétricas e de gestação atual, no qual as perguntas eram lidas pelo pesquisador com seus devidos esclarecimento sobre cada questão e as respostas eram registradas no questionário ao longo da ligação.

As ligações eram realizadas na segunda-feira, quarta-feira e sexta-feira, no período das 16 às 18 horas, no qual eram contatadas três gestantes por dia. Não foram realizados contatos telefônicos em finais de semana ou feriados.

Além da possibilidade das ligações telefônicas, foi realizada coleta de dados durante dois encontros do grupo de gestantes de uma das equipes da manhã cujas reuniões são realizadas uma vez ao mês nas sextas-feiras pela manhã no horário das 8 às 11 horas. Foi possível coletar 10 questionários em dois encontros do grupo.

O presente plano de trabalho é vinculado ao subprojeto "Morbidades em Porto Velho, constitutivo do projeto matriz intitulado "Estudo sobre morbidades em Rondônia: a assistência, a formação e o ensino em discussão", aprovado pelo CEP/UNIR, CAAE 85533318.9.0000.5300, sob o parecer n. 2.548.115. 


\section{RESULTADOS}

Constatou-se que de 39 gestantes, a maioria possui idade entre 15 e 19 anos (56,41\%) com média de idade de $(20,06 \pm 2,95)$ anos, se autodeclaravam pretas ou pardas $(76,92 \%)$ e com estado civil casadas ou em união estável (82,05\%).

Quanto ao grau de escolaridade o maior percentual foi de gestantes com ensino fundamental $(46,15 \%)$, em que $(74,36 \%)$ indicaram renda familiar que não chegavam a um salário-mínimo e $(76,92 \%)$ não possuíam nenhuma atividade remunerada (Tabela 1).

Tabela 1 - Perfil sociodemográfico e econômico de gestantes entre 10 e 24 anos acompanhadas por equipes de saúde da família matutinas de uma Unidade de Saúde da Família.

\begin{tabular}{|c|c|c|}
\hline Variáveis & $\mathbf{n}$ & $\%$ \\
\hline \multicolumn{3}{|l|}{ Idade } \\
\hline 15 a 19 anos & 22 & 56,41 \\
\hline 20 a 24 anos & 17 & 43,59 \\
\hline \multicolumn{3}{|l|}{ Cor da pele } \\
\hline Branca & 6 & 15,38 \\
\hline Parda/preta & 30 & 76,92 \\
\hline Amarela & 3 & 7,69 \\
\hline \multicolumn{3}{|l|}{ Estado civil } \\
\hline Solteira & 7 & 17,95 \\
\hline Casada/União estável & 32 & 82,05 \\
\hline \multicolumn{3}{|l|}{ Morando com o companheiro } \\
\hline$<1$ ano & 14 & 35,90 \\
\hline 1 a 4 anos & 15 & 38,46 \\
\hline 5 a 9 anos & 3 & 7,69 \\
\hline Sem parceiro & 7 & 17,95 \\
\hline \multicolumn{3}{|l|}{ Escolaridade } \\
\hline Ensino fundamental incompleto & 18 & 46,15 \\
\hline Ensino fundamental completo & 1 & 2,56 \\
\hline Ensino médio incompleto & 11 & 28,21 \\
\hline Ensino médio completo & 9 & 23,08 \\
\hline \multicolumn{3}{|l|}{ Renda familiar } \\
\hline$<1$ salário & 29 & 74,36 \\
\hline 1 a 3 salários & 8 & 20,51 \\
\hline Nenhuma & 2 & 5,13 \\
\hline \multicolumn{3}{|l|}{ Ocupação atual } \\
\hline Empregada, não servidora pública & 3 & 7,69 \\
\hline Do lar & 6 & 15,38 \\
\hline Sem ocupação & 30 & 76,92 \\
\hline Total & 39 & 100,00 \\
\hline
\end{tabular}

Fonte: Rodrigues MAS, et al., 2022. 
Ao serem questionadas a respeito das práticas sexuais, a maioria das gestantes se tornou sexualmente ativa na faixa etária dos 15 aos 19 anos (53,85\%). Em relação à prevenção de Infecções Sexualmente Transmissíveis (IST) (82,05\%) relataram preocuparem-se com o risco para contaminação e $(53,85 \%)$ afirmaram a utilização de métodos de barreira como forma de prevenção das IST em algum momento. Quando questionadas sobre o uso do preservativo na última relação sexual $87,18 \%$ referiram não ter feito uso de tal método na última relação sexual (Tabela 2).

Sobre as orientações fornecidas sobre uso de métodos anticoncepcionais e orientações recebidas de profissionais de saúde, $64,10 \%$ afirmaram receber tais orientações. Com relação ao número de parceiros sexuais no último ano, 84,62\% assentiram ter somente um único parceiro sexual (Tabela 2).

Tabela 2 - Perfil das práticas sexuais e reprodutivas das gestantes com idade de 10 a 24 anos, acompanhadas pelas equipes de Saúde da Família matutinas de uma unidade de Saúde da Família.

\begin{tabular}{lcc}
\hline Variáveis & $\mathbf{n}$ & $\%$ \\
\hline Início das relações sexuais & 18 & 46,15 \\
\hline 10 a 14 anos & 21 & 53,85 \\
15 a 19 anos & 32 & \\
\hline Preocupação sobre a prevenção de ISTs & 72,05 \\
\hline Sim & & 17,95 \\
Não & 21 & \\
\hline Utilização de método de barreira para prevenção de ISTs & 53,85 \\
\hline Sim & 18 & 46,15 \\
Não & & \\
\hline Orientação de profissional de saúde sobre o uso de métodos contraceptivos & 64,10 \\
\hline Sim & 25 & 35,90 \\
Não & 14 & \\
\hline Uso de preservativo na última relação sexual & & 12,82 \\
\hline Sim & 5 & 87,18 \\
Não & 34 \\
\hline Número de parceiros sexuais no último ano & & \\
\hline Um & 33 & 84,62 \\
Dois & 4 & 10,26 \\
Três ou mais & 2 & 5,13 \\
\hline Total & 39 \\
\hline Fonte: Rodrigu & 100,00 \\
\hline
\end{tabular}

Fonte: Rodrigues MAS, et al., 2022.

Com relação às características da gestação atual constatou-se que 92,31\% das gestações não foram planejadas, porém, $(97,44 \%)$ relataram ter recebido apoio familiar. Na descoberta da gestação $(53,85 \%)$ buscaram a unidade para saber se estava gestante, $(53,85 \%)$ tiveram o início do pré-natal entre a sexta e a décima segunda semana de gravidez. Desse modo, 100\% afirmaram ser acompanhadas pelo enfermeiro, $74,36 \%$ pelo médico da equipe e pouco se observou o acompanhamento pelo odontólogo (17,95\%) e ACS das equipes em que somente $10,26 \%$ relatou acompanhamento pelos agentes (Tabela 3).

Observou-se que $(33,33 \%)$ faziam acompanhamento em serviço de referência para gestação de alto risco e desse modo, quando questionadas a respeito sobre a busca por algum estabelecimento de saúde de referência em casos de emergências obstétricas ou intercorrências clínicas $(53,85 \%)$ afirmaram a busca por tais serviços, até o presente momento (Tabela 3). 
Tabela 3 - Perfil da gestação atual das gestantes de 10 a 24 anos acompanhadas pelas equipes de Saúde da Família matutinas de uma unidade de Saúde da Família.

\begin{tabular}{|c|c|c|}
\hline Variáveis & $\mathbf{n}$ & $\%$ \\
\hline \multicolumn{3}{|c|}{ Planejamento da gestação atual } \\
\hline Sim & 3 & 7,69 \\
\hline Não & 36 & 92,31 \\
\hline \multicolumn{3}{|c|}{ Rede de apoio durante a descoberta da gestação } \\
\hline Sim & 38 & 97,44 \\
\hline Não & 1 & 2,56 \\
\hline \multicolumn{3}{|c|}{ Busca da USF para diagnóstico da gestação } \\
\hline Sim & 18 & 46,15 \\
\hline Não & 21 & 53,85 \\
\hline \multicolumn{3}{|c|}{ Idade gestacional na primeira consulta } \\
\hline$<6$ semanas & 5 & 12,82 \\
\hline 6 a 12 semanas & 21 & 53,85 \\
\hline 12 a 20 semanas & 10 & 25,64 \\
\hline 21 a 28 semanas & 3 & 7,69 \\
\hline \multicolumn{3}{|c|}{ Profissional que acompanhou o pré-natal } \\
\hline Enfermeiro & 39 & 100,00 \\
\hline Médico & 29 & 74,36 \\
\hline Dentista & 7 & 17,95 \\
\hline ACS & 4 & 10,26 \\
\hline \multicolumn{3}{|c|}{ Procura por serviços de referência } \\
\hline Sim & 21 & 53,85 \\
\hline Não & 18 & 46,15 \\
\hline \multicolumn{3}{|c|}{ Acompanhamento de pré-natal de alto risco } \\
\hline Sim & 13 & 33,33 \\
\hline Não & 26 & 66,67 \\
\hline Total & 39 & 100,00 \\
\hline
\end{tabular}

Fonte: Rodrigues MAS, et al., 2022.

Dentre as principais intercorrências relatadas, os três mais frequentes foram intercorrências do trato gastrointestinal (náuseas e vômitos) $(74.36 \%)$, seguidos por problemas do trato urinário (principalmente infecção bacteriana) $(64,10 \%)$ e as queixas ginecológicas $(58,97 \%)$. Com relação à hipertensão arterial e diabetes melitus pouco se observou a frequência desses problemas clínicos entre as gestantes (Tabela 4).

Tabela 4 - Principais problemas e intercorrências clínicas durante o período gestacional das gestantes acompanhadas pelas equipes de Saúde da Família matutinas de uma unidade de Saúde da Família.

\begin{tabular}{lcc}
\hline Queixas ou problemas gestacionais & $\mathbf{n ~ = 3 9}$ & $\%$ \\
\hline Problemas gastrointestinais & 29 & 74,36 \\
Problemas de trato urinário & 25 & 64,10 \\
Problemas ginecológicos & 23 & 58,97 \\
Cefaleia, tontura, desmaios, cansaço ou indisposição & 21 & 53,85 \\
Problemas tegumentares & 20 & 51,28 \\
Infecção sexualmente transmissível (sífilis) & 5 & 12,82 \\
Violência & 4 & 10,26 \\
\hline
\end{tabular}

Fonte: Rodrigues MAS, et al., 2022. 


\section{DISCUSSÃO}

Estudos apontam que a faixa etária em que mais ocorrem gestações em adolescente encontra-se entre 16 e 19 anos e os principais motivos para esses eventos são a falta de acesso a serviços de saúde, pouco conhecimento e empoderamento acerca das políticas de planejamento reprodutivo e ausência de educação sexual (SABINO KCV, et al., 2019; SOARES IA e SILVA BA, 2020).

Com relação étnica e racial o estudo revelou que a maior parte das gestantes que realizavam pré-natal se autodeclaravam como pardas e pretas. Theophilo RL, et al. (2018), afirmam que as mulheres que buscam assistência pré-natal de cor preta ou parda aparecem nas piores condições socioeconômicas e de acesso à rede de saúde, caracterizando-as em situação de vulnerabilidade, apontando para iniquidades e desigualdades, mesmo dentro do SUS, como os resultados encontrados nesta pesquisa.

No perfil social dessas mulheres, a baixa escolaridade evidenciou-se com poucos anos de estudo visto que a maior parte referiu ensino fundamental incompleto. Considerando que grande parte das gestantes se encontram na faixa etária de 15 até 19 anos, já deveriam estar concluindo o ensino médio. Um estudo realizado por Fernandes RMF, et al. (2015), em uma capital do nordeste brasileiro, apontou que um importante motivo de abandono dos estudos foi a gestação, considerado o grande fator para a interrupção da vida escolar.

Um estudo realizado por Sabino KCV, et al. (2019), aponta que a maior parte dessas gestantes que se encontram ou buscam assistência pré-natal na APS, vivem com rendas menores que um salário-mínimo, em sua grande maioria, a fonte de renda única provém do cônjuge /parceiro ou mesmo da família, quando está ainda se encontra morando com os pais.

Com relação às práticas sexuais e reprodutivas, observa-se nesse estudo que a maior parte das gestantes teve o início de suas vidas sexuais entre 15 e 19 anos e suas gestações no mesmo período. Alguns estudos demonstram que há uma associação entre o início da vida sexual em menores condições socioeconômicas e menor grau de escolaridade com as maiores chances de engravidar logo na primeira relação sexual (CRUZ MS, et al., 2016; DIAS BF, et al., 2020).

Salienta-se, ainda, que a redução ou o estigma sobre a educação sexual familiar e escolar se configura como fator precursor para gestação não planejada entre jovens em contexto culturais diversos, o qual é potencializado quando distante de ambientes educacionais ou possui sua vida escolar interrompida (SILVA FN, et al., 2012; FERNANDES DER, et al., 2020; CINTRA LCG, et al., 2020).

Outro fator observado foi que boa parte das gestantes têm preocupação quanto à prevenção de IST e relatam conhecer a formar de uso do preservativo, porém quando questionadas acerca do uso em seu relacionamento atual, não é observado adesão do método. Tal fator é evidenciado pela literatura como uma questão subjetiva dessas mulheres devido à confiança no parceiro ou mesmo no fato de estarem em um relacionamento estável com uma única pessoa (COSTA GF, et al., 2018; SANTOS SC, et al., 2018).

Ademais, muitas dessas mulheres relatam ter apenas um parceiro ao longo da vida sexual e da gestação, o que subsidia a escolha pela não adesão. O uso dos métodos de barreiras são aceitáveis apenas para a evitabilidade de gravidez não desejada e pouco reconhecidos como meios de redução do risco sexual dessas populações (CRUZ MS, et al., 2016; MARANHÃO TA, et al., 2017).

A prevenção da gestação e o sexo seguro não se limitam ao uso de preservativo, mas a existência de desenvolvimento de habilidades afetivas na construção da infância e juventude, a fim de que o jovem/adolescente possa compreender os limites de seus desejos sobre o outro, durante seu processo de formação individual (CABRAL CS e BRANDÃO ER, 2020).

Os achados desse estudo revelam que grande parte das gestações ocorreram de forma não intencional. Pode-se inferir que os comportamentos sexuais de risco como a não adesão ao preservativo de barreira em razão dos relacionamentos com um único parceiro aumentam as chances de gestações não planejadas entre jovens e adolescentes. Estudos apontam a falta de orientação acerca dos riscos a não adesão de medidas anticoncepcionais durante a gestação, problema evidenciado nos serviços de saúde e na APS, o que demonstra déficits na qualidade de assistência ofertada a esse público (SILVA ELC, et al., 2014; SANTOS ACF, et al., 2020). 
É observado em diversas pesquisas realizadas com indivíduos nessa faixa etária que, em razão das grandes mudanças inerentes a esse período de transição para a vida adulta, a descoberta da sexualidade é um grande marco para o jovem, entretanto, o início das relações sexuais desassociadas da educação sexual e planejamento reprodutivo, configuram o cenário para o aumento das gestações indesejadas e os casos IST entre pessoas jovens (OKUDA GT, et al., 2017; DIAS BF, et al., 2020).

Outra inferência que se faz com base nesses achados é que a descoberta e confirmação da gravidez ocorrem em estabelecimentos privados de saúde, porém o seguimento pré-natal se dá majoritariamente pelo SUS, no âmbito da APS/ESF, evento esse que ocorre principalmente nas regiões Norte e Nordeste são. Já em regiões mais desenvolvidas tal cenário ser comporta de forma distinta com maiores números de consultas ao longo do período gestacional no âmbito da APS e do SUS (QUEIROZ MVO, et al., 2014; CRUZ MS, et al., 2016).

Outrossim, os impactos da descoberta da gestação não planejada são de extrema relevância científica, uma vez que tal acontecimento repercute não somente na vida da mulher, como do parceiro e de suas famílias. Os fatores psicossociais enfrentados por essas jovens gestantes são imersos em sentimentos como rejeição, tristeza e angústias advindos não somente da descoberta da gravidez, mas dos conflitos familiares devido ao evento e aos julgamentos sociais do meio em que vivem (SILVA ELC, et al., 2014; COSTA GF, et al., 2018).

Com relação ao início do pré-natal a pesquisa revelou que quase metade das gestantes teve a primeira consulta depois do primeiro trimestre de gestação ou não comparecimento as consultas. Diversos fatores contribuem para diagnóstico tardio da gestação, dentre eles, o contexto cultural em que essas meninas estão inseridas, mas principalmente as dificuldades de acesso aos serviços de saúde, demonstrando que só se tem conhecimento da população jovem sexualmente ativa no território quando esta busca por esses serviços de acompanhamento gestacional (VIELLAS EF, et al., 2014; RODRIGUES VHV, et al., 2020).

Com relação à assistência recebida pelos profissionais das ESF, verificou-se um predomínio do acompanhamento pelo enfermeiro nos cuidados pré-natais, o qual se torna da referência para essas usuárias. Entretanto o desempenho de uma assistência integral só é possível de ser alcançado com a participação de todos os profissionais presentes na ESF. É apontado na literatura que a assistência prestada pelos profissionais na APS/ESF se pautam na sobreposição de protocolos e rotinas em detrimento das singularidades trazidas por essas mulheres, distanciando-se da integralidade e negando a essas gestantes serem sujeitos de direito (MELO MCP e COELHO EAC, 2011; SANTOS ACF, et al., 2020).

Outra questão importante demonstrada na pesquisa foi o baixo acompanhamento realizado pelos ACS ao longo da gestação, uma vez que é imprescindível o papel desses profissionais no território, visto que a sua inserção facilita não somente captação dessas jovens futuras mães, mas impacta diretamente na adesão dessas mulheres ao pré-natal. Além disto, facilita a formação do vínculo, a fim de sanar as demandas de saúde, a observação das intercorrências clínicas fora do consultório e auxílio no período puerperal, aleitamento materno e planejamento reprodutivo (OLIVEIRA MP, et al., 2015).

Outro achado acerca do pré-natal dessas meninas revela o baixo índice de realização do acompanhamento gestacional pelo odontólogo. Estudo realizado por Júnior DJK, et al. (2019), apontam que os menores índices de acompanhamento pelo odontólogo durante o pré-natal são em mulheres jovens (menores de 20 anos). Em revisão integrativa realizada por Silva CC, et al. (2020), foi apontado a baixa adesão dessa população para esse tipo de serviço, visto a pouca significância que essas meninas possuem acerca da saúde bucal e não reconhecimento da equipe de saúde acerca do papel do dentista na prestação de cuidados pré-natais.

Quando questionadas a respeito das intercorrências clínicas observou-se que um grande percentual relatou a necessidade de buscar por serviços de emergência por queixas clínicas. Apesar da maior parte dos nascimentos de filhos de mães adolescentes e jovens ocorram por via vaginal ainda são relevantes às taxas de eventos relacionados à intercorrência clínicas ou obstétricas como o aumento de curvas glicêmicas e síndromes hipertensivas na gestação (CRUZ MS, et al., 2016; RIBEIRO JF, et al., 2017; DIAS BF, et al., 2020). 
$\mathrm{Na}$ realização desta pesquisa observou-se que as maiores queixas ou intercorrências estiveram relacionadas aos problemas gastrointestinais, do trato urinário, ginecológicos, tegumentares e sintomas mais inespecíficos (cefaleia, tontura, desmaios, cansaço ou indisposição). Tais achados são observados na literatura como as principais intercorrências ao longo do acompanhamento pré-natal na atenção primária, visto que a atenção a tais problemas se faz necessária devido ao agravante das vulnerabilidades correlacionadas a idade, levando a complicações materno-fetais (AZEVEDO WF, et al., 2015; FERNANDES RFM, et al., 2018).

Silva CC, et al. (2020), constataram que os problemas de trato urinário e os ginecológicos estão entre as maiores queixas e motivos de buscas por serviços de referências ao longo da gestação. São observados como os mais graves que podem levar a desdobramentos negativos sobre a saúde dessas mulheres, assim como contribuem para o aumento da mortalidade materna (RIBEIRO JF, et al., 2017).

\section{CONCLUSÃO}

O perfil de adolescentes e jovens gestantes, encontrado neste estudo foi constituído majoritariamente por meninas de 15 a 19 anos, de raça cor preta ou parda, a grande maioria abandou os estudos, não possui ocupação e vive com rendas inferiores a um salário-mínimo, morando junto ao parceiro. Ao se observar o histórico sexual e reprodutivo dessas jovens mulheres, se constata que a maior parte possui práticas sexuais de risco para sua saúde e da criança e não faz adesão à utilização de camisinha ou qualquer outro método de barreira nas relações sexuais durante a gestação. Recomenda-se enfatizar a importância da atenção integral ao binômio, particularmente, pelo ACS. Além disto, as orientações centradas nas necessidades das gestantes são fundamentais para prevenção de complicações e intercorrências na gestação.

\section{REFERÊNCIAS}

1. AZEVEDO WF. Complicações da gravidez na adolescência: revisão sistemática da literatura. Einstein, 2015; 13(4): 618-26.

2. CRUZ MS, et al. Perfil socioeconômico, demográfico, cultural, regional e comportamental na gravidez na adolescência no Brasil. Planejamento e politicas publicas, 2016; (46): 244-66.

3. CINTRA LCG. et al. Panorama do perfil sociodemográfico e cultural da adolescente grávida. Braz. J. of Develop., 2020; 6(11): 92464-74.

4. COSTA GF, et al. Fatores psicossociais enfrentados por grávidas na fase final da adolescência. Rev Bras Promoç Saúde, 2018; 31(2):1-8.

5. CABRAL CS, BRANDÃO ER. Gravidez na adolescência, iniciação sexual e gênero: perspectivas em disputa. Cad Saúde Pública, 2020; 36(8): 1-5.

6. DIAS BF, et al. Perfil clínico e epidemiológico da gravidez na adolescência: um estudo ecológico. Arq. Catarin. Med., 2020; 49(1): 10-23.

7. FERNANDES RMF, et al. Características do pré-natal de adolescentes em capitais das regiões sul e nordeste do brasil. Texto Contexto Enferm, 2015; 24(1): 80-6.

8. FERNANDES RFM, et al. Intercorrências obstétricas que ocorrem durante a gravidez na adolescência. Cienc Cuid Saude, 2018; 17(1): 1-7.

9. FERNANDES DER, et al. Produção científica de Enfermagem sobre gravidez na adolescência: revisão integrativa. Aquichan, 2020; 20(2): e2025.

10. JACOB DSG, et al. Gravidez na adolescência: uma análise dos determinantes sociais. Braz J of Develop, 2020; 6(2): 8080-8.

11. JUNIOR DJK, et al. Não realização de consulta odontológica entre gestantes no extremo sul do Brasil: um estudo de base populacional. Ciência \& Saúde Coletiva, 2019; 24(10): 3889-96.

12. KASSAR SB, et al. Comparações das condições socioeconômicas e reprodutivas entre mães adolescentes e adultas jovens em três maternidades públicas de Maceió, Brasil. Rev Bras Saúde Matern Infant, 2006; 6(4): 397-403.

13. MARANHÃO TA, et al. Repercussão da iniciação sexual na vida sexual e reprodutiva de jovens de capital do Nordeste brasileiro. Cienc Saude colet, 2017; 22(12): 4083-93.

14. MARTINS MMF, et al. Acesso aos serviços de saúde de Atenção Primária à Saúde por adolescentes e jovens em um município do Estado da Bahia, Brasil. Cad Saúde Publica, 2019; 35(1): e00044718. 
15. MINISTÉRIO DA SAÚDE. Secretaria de Atenção à Saúde. Departamento de Ações Programáticas e Estratégicas. Proteger e cuidar da saúde de adolescentes na atenção básica. Brasília - DF: Brasil, 2018. Disponível em: https://bvsms.saude.gov.br/bvs/publicacoes/proteger_cuidar_adolescentes_atencao_basica_2ed.pdf. Acessado em: 15 de fevereiro de 2022.

16. MINISTÉRIO DA SAÚDE. Secretária de Atenção em Saúde. Departamentos de Ações Programáticas Estratégicas. Diretrizes nacionais para a atenção integral à saúde de adolescentes e jovens na promoção, proteção e recuperação da saúde. Brasília - DF: Brasil, 2010. Disponível em: https://bvsms.saude.gov.br/bvs/publicacoes/proteger_cuidar_adolescentes_atencao_basica.pdf. Acessado em: 15 de fevereiro de 2022.

17. MELO MCP, COELHO EAC. Integralidade e cuidado a grávidas adolescentes na Atenção Básica. Cienc Saúde Coletiva, 2011; 16(5): 2549-58.

18. PLUMMER ML, et al. Global Accelerated Action for the Health of Adolescents (AA-HA!): guidance to support country implementation. Geneva: (c) World Health Organization, 2017.

19. OKUDA GT, et al. Perfil social e obstétrico de gestantes adolescentes. Cienc Cuid Saúde, 2017; 6(2): 1-8.

20. OLIVEIRA MP, et al. Cuidado às adolescentes grávidas: perspectiva e atuação de agentes comunitários de saúde. Rev Enferm UERJ, 2015; 23(1): 76-81.

21. QUEIROZ MVO, et al. Perfil da gravidez na adolescência e ocorrências clínico-obstétricas. Rev Rene, 2014; 15(3): 455-62.

22. RIBEIRO JF, et al. Complicações obstétricas em adolescentes atendidas em uma maternidade pública de referência. Rev Enferm UFPE, 2017; 11(7): 2728-35.

23. RODRIGUES ARM, et al. Gravidez de alto risco análise dos determinantes de saúde. Sanare, 2017 ; 16 (supl): $23-8$.

24. RODRIGUES VHV. Fatores associados ao início tardio do pré-natal: revisão integrativa. Medical Science Nursing, 2020; 1 (esp): 76-87.

25. SANTOS SC, et al. Perfil das gestantes adolescentes no interior de Pernambuco. Rev Bras Educ Saúde, 2018; 8(3): 49-53.

26. SANTOS ACF, et al. Abordagem do Enfermeiro na Gravidez na Adolescência. Braz. J. of Develop., 2020; 3(6): 17438-56.

27. SILVA FN, et al. Gravidez na adolescência: perfil das gestantes, fatores percussores e riscos associados. Revista Eletrônica Gestão \& Saúde, 2012; 3(3): 844-96.

28. SILVA ELC, et al. Gravidez e dinâmica familiar na perspectiva de adolescentes. Acad. Paul. Psicol., 2014; 34(86): 118-38.

29. SILVA MRB, et al. Por que elas não usam?: Um estudo sobre a não adesão das adolescentes aos métodos contraceptivos e suas repercussões. Saúde em Redes, 2019; 1(4): 75-83.

30. SILVA CC, et al. Acesso e utilização de serviços odontológicos por gestantes: revisão integrativa de literatura. Ciência \& Saúde Coletiva, 2020; 25(3): 827-835.

31. SABINO KCV, et al. Perfil sociodemográfico e comportamental de gestantes adolescentes de uma maternidade de referência. Revista de Enfermagem UFPI, 2019; 8(2): 10-7.

32. SOARES IA, SILVA BA. Gravidez na adolescência: perfil sociodemográfico da $8^{\underline{a}}$ regional de saúde do sudoeste do paraná no período de 2015 a 2018. Acta Elit Salutis, 2020; 2(1): 15.

33. THEOPHLO RL, et al. Vulnerabilidade de mulheres negras na atenção ao pré-natal e ao parto no SUS: análise da pesquisa da Ouvidoria Ativa. Ciência \& Saúde Coletiva, 2018; 23(11): 3505-16.

34. VIELLAS EF, et al. Assistência pré-natal no Brasil. Cad. Saúde Pública, 2014; 30(Supl.1): 85-100. 